\title{
Research on the Planning of Attached Green space in Residential area from the perspective of elderly activities
}

\author{
Xiangjun Dai* \\ College of Architecture and Art, Central South University, Chang Sha, Hu Nan, China
}

\begin{abstract}
The aging of population in China has been further deepened, and the living environment of the elderly has been widely concerned. As an important place for residents' activities, the attached green space of residential area plays an important role in optimizing urban ecological environment, shaping urban landscape, providing recreation services and preventing and avoiding disasters. At present, the landscape space design of the attached green space in the residential area specially designed for the elderly is not in harmony with its demand intensity. Scientific planning of the attached green space in the residential area can enrich the daily recreational activities needed by the elderly, so as to better improve the quality of life of the elderly and promote their health. From the behavior characteristics of the elderly, the activities of the affiliated green space of residential demand, put forward under the background of an aging society residential affiliated green space reasonable planning direction, for the effective supply of the elderly daily behavior space, sufficient to guide the healthy life of the elderly to improve life quality and health level put forward the corresponding design strategy, try for the urban residential affiliated green space elements, structure, system function coordination optimization to provide theoretical support.
\end{abstract}

\section{Introduction}

With the continuous acceleration of the aging process of China's population, the aging of China's population has reached its peak in advance. Faced with this serious problem, the society needs to pay attention to the environmental health of the elderly and provide them with more scientific and appropriate activity places to meet their living needs [1]. As the elderly grow in age, their physiological functions begin to decline. When planning the attached green space in residential areas, the characteristics of the elderly should be fully considered and the specific needs of the elderly should be integrated into the whole design, so as to better meet the requirements of the activities of the attached green space in residential areas [2]. In addition, the design of green space functional zoning should also conform to the differences in the behavior and activities of the elderly, so as to provide more recreation and leisure space for the elderly and enable them to better participate in social activities. This paper studies the action mechanism of attached green space in residential area in elderly activities from the perspectives of psychological regulation and physiological health.

\section{Analysis of the characteristics of the elderly related to the activities of attached green space in residential areas}

\subsection{Classification of the elderly}

This paper defines the population aged 60 and above as the elderly. According to the "Architectural Design Code for the Elderly", the elderly are specifically divided into three categories: self-care elderly, assisted elderly and nursing elderly, as shown in Table 1.

Table1. Classification of the elderly

\begin{tabular}{cll}
\hline \multicolumn{1}{c}{ Classification } & \multicolumn{1}{c}{ Definition } & \multicolumn{1}{c}{ Activity status } \\
\hline self-care elderly & $\begin{array}{l}\text { Elderly people who can take care of } \\
\text { themselves }\end{array}$ & $\begin{array}{l}\text { Go to the green space attached to the } \\
\text { residential area alone for activities }\end{array}$ \\
assisted elderly & $\begin{array}{l}\text { Elderly people who need assistance from } \\
\text { auxiliary facilities }\end{array}$ & $\begin{array}{l}\text { Go to the green with the help of your children } \\
\text { or a caregiver }\end{array}$ \\
nursing elderly & $\begin{array}{l}\text { Elderly people whose lives depend on the } \\
\text { care of others }\end{array}$ & $\begin{array}{l}\text { The activity of attached green space in } \\
\text { residential area is relatively less }\end{array}$ \\
\hline
\end{tabular}

*Corresponding author’s e-mail: 1242736383@qq.com 


\subsection{Physiological characteristics of the elderly}

With the increase of age, the physiological function of the elderly has obvious changes, mainly manifested as physical strength decline, sleep time reduction, physical ability degradation, mobility. The physiological function changes of the elderly make the elderly have higher requirements for the use of the attached green space in residential areas, and it is necessary to combine the special needs of the elderly with the design of the attached green space in residential areas to provide suitable places for the activities of the elderly [3].

\subsubsection{Sensory degradation}

Humans need to experience a minimum threshold to receive information from the outside world, and that threshold expands with age. Therefore, when the elderly get external information, they need to be exposed to more information in order to get the corresponding perception. When the elderly move around in the attached green space of residential area, they will show a slow adaptation and inefficient understanding of the environment.

\subsubsection{Visual deterioration}

The elderly's ability to accept light continues to weaken, and their perception of light and adaptability to darkness have declined. Their outstanding visual feature is that they are more likely to see warm colors such as yellow, orange, and red, compared to other cool colors. Perception ability declines.

\subsubsection{Deterioration of hearing, taste and smell}

The elderly have certain difficulties in distinguishing high and low sounds, and it is difficult to hear the content of the conversation even in a low decibel environment. The change of hearing affects the communication between the elderly and the outside world. At the same time, the elderly with hearing loss also have corresponding safety risks during activities. With the increase of age, the sense of taste and smell of the elderly are gradually degraded. At the same time, the elderly are relatively slow to respond to pain, their body balance ability is reduced, and accidental injuries are prone to occur during exercise.

\subsubsection{Psychological changes of the elderly}

The psychological burden of the elderly continues to increase. The weakening of physical functions is accompanied by psychological difficulties. The psychological emotions such as loneliness, loss, loneliness and unsatisfactory desire to communicate aggravate the psychological burden of the elderly. However, with the lifestyle and thinking with the change of concept, the elderly are also actively seeking solutions while producing negative psychological activities. This makes the elderly more proactively involved in the whole life, making life more dynamic, and thus further promoting the use efficiency of the auxiliary green space in the residential area is improved.

\subsubsection{Behavioral characteristics of the elderly}

With the increase of age, the behavioral characteristics of the elderly change more obviously, which are mainly reflected in the slow response, the gradual decline in the speed and efficiency of action, and the weaker execution ability to deal with complex behaviors. The elderly will become more cautious in the process of activities. The elderly need longer time to react to complex things in the outside world, and the elderly are more inclined to congregate activities when they go out. Time and location are obviously fixed, and the elderly are used to doing it in familiar locations. Activities, more willing to communicate in a familiar environment.

\section{The special needs of the elderly for the green space activities in the residential area}

When planning and designing the auxiliary green space in the residential area, it should conform to the living habits of the elderly, and provide comfortable and safe activity places for the elderly. First of all, the safety of the place must be ensured. Due to the growth of age, the elderly will have difficulty in moving. During the activity, they will rub against the discordant factors of the place, which will cause damage to the body. Secondly, starting from the psychological aspect, the elderly are weak in adapting to complex environments and will have a sense of rejection in the course of activities. The design needs to be friendly to meet the psychological dependence needs of the elderly [4]. Due to their psychological and physical characteristics, the elderly are more sensitive to spatial layout. In the process of designing the auxiliary green space in the residential area, comfort needs to be considered, including the comprehensive consideration of space size, location, color and environment. The design of the auxiliary green space in the residential area also needs to consider its functionality, such as physical exercise, chat, and entertainment facilities. At the same time, it is also necessary to consider its ease of use. As the elderly move slowly, in the design process, try to simplify the design as much as possible to provide a convenient place for activities.

The elderly's ability to adapt to the external environment has gradually decreased, and various common senile diseases such as respiratory diseases, diabetes, and hearing loss have begun to appear. Most of these senile diseases are caused by environmental problems. Judging from the current urban development status, the sanitary environment This is the most worrying issue. Smog and sandstorms have greatly restricted the travel of the elderly. These environmental problems can be scientifically improved by relying on the attached green space in residential areas. The design of ancillary green space in residential areas should take into account the needs of the elderly in green space activities as well as urban environmental governance. When the elderly go out 
for activities, due to the limitation of receptor energy, they will not engage in strenuous activities, usually walking and jogging, and the range of activities of the elderly is relatively fixed, so they are more sensitive to the conditions of the scenery in the space [5]. The elderly like places leeward and well ventilated, and the design of the auxiliary green space in the residential area needs to consider the activity preferences and physical health of the elderly.

\section{Planning and design strategy of auxiliary green space in residential area based on the activity requirements of the elderly}

\subsection{Green space needs to reach a reasonable intensity of demand}

In the design process of residential area subsidiary green space, avoid designing multiple smaller and independent spaces. Such space service facilities are not perfect, can accommodate a small number of users, and cannot meet the needs of more elderly people to participate in entertainment activities together. [6]. In addition, a single green space is easily disturbed by the external environment, and the elderly cannot participate in the activity in a comfortable state. Therefore, when designing the auxiliary green space in the residential area, try to design a large public space with a certain scale.

\subsection{Scientifically consider the actual situation from multiple angles}

When designing ancillary green spaces in residential areas, first consider factors such as land area, surrounding land use conditions, the needs of the elderly, and other residents' needs; secondly, consider the local climate environment and topography, and rationally arrange different types of spatial layout; and also consider the scope of services The population of the elderly in China, the characteristics of age distribution, and the needs of activities. In order to design a comfortable green space according to local conditions, the site needs can be summarized and analyzed through field surveys, questionnaires, interviews and other methods [7], and a reasonable design plan can be proposed. The physical and psychological needs of the elderly are different from those of other age groups. The design can introduce related theories of psychology, outdoor recreation, and environmental behavior. After comprehensively considering various factors, the building has a variety of functions, safety, comfort, and fullness. The green space attached to the flexible residential area with humane care.

\subsection{Spatial classification of auxiliary green space in residential areas based on the activities of the elderly}

Different old people have their own preferences, so it is necessary to design corresponding activity venues according to their psychological characteristics. The article divides the activity space into dynamic space and static space. Dynamic venues mainly host fitness, ball games, dance, martial arts, long whip and other activities. For dynamic event venues, it is necessary to ensure sufficient area, set up shelters around the venue, and at the same time, the surrounding venues should be as flat as possible to avoid excessive height differences in the venue, so as to avoid potential dangers to the activities of the elderly. In the design process, the rest service facilities should also be perfected. Benches, public toilets, coat storage, etc. need to be reasonably arranged, taking full account of the various situations that the elderly may appear in activities [8]. The setting of rest facilities allows the elderly to enjoy the landscape, relax, and chat during the rest, creating a quiet and comfortable rest space for the elderly.

During the activities of the elderly, there are corresponding static activities. In the dynamic and static activity areas, transition areas should be designed to better ensure the quality of leisure and entertainment for the elderly. In static activities, the elderly mainly play chess, sit quietly, and enjoy flowers. This type of activity produces less noise and less activity. Static activities can be both outdoors and indoors. Therefore, a corresponding leisure area can be set up outdoors, so that more elderly people with the same hobbies can gather together to meet their entertainment needs. For indoor activities, it is necessary to provide a spacious and bright venue[9]. In the venue, table tennis tables and chess tables can be appropriately placed. In the design of urban green space, a certain area of lake water body can be set up, which is conducive to maintaining the ecological balance in the area, and the elderly can avoid various diseases caused by dryness during exercise. The elderly can also go fishing to enrich the content of outdoor activities.

\section{The mechanism of the green space attached to the residential area on the activities of the elderly}

Cities are developing more and more rapidly, and the destruction of the environment is gradually intensifying. People's desire for the natural environment is increasing. More and more elderly people choose to go out of their homes for outdoor activities. With the improvement of specific elderly public service facilities, Urban green space will gradually become the main place for elderly activities. In the process of outdoor space activities, the elderly can cultivate interest in recreation, promote social interaction, and enhance self-satisfaction. The ancillary green space in the residential area can not only provide entertainment and leisure places for the elderly, but also the related activities carried out on the places are of great help to the physical and mental health of the elderly. The article will show the mechanism of action of residential green space on the activities of the elderly from the following three aspects. 


\subsection{Meet the psychological needs of the elderly}

With the increase of age, most elderly people choose to retire after the age of 60 . They used to be the main source of the family's economy. They have become elderly people with ample free time. The huge psychological gap makes the elderly feel uncomfortable. Willing to accept this status quo. The construction of ancillary green space in the residential area can provide more leisure and entertainment places for the elderly. The elderly can talk with their peers to enjoy the body and mind and reduce the occurrence of depression. Set up an interactive entertainment zone for the elderly in the green space attached to the residential area, allowing the elderly to carry out various cultural and entertainment activities in this area, continuously expanding the interests of the elderly, and promoting the social activities of the elderly, which can better keep the elderly Happy mood [10]. The elderly actively participate in the entire cultural and entertainment activities, so that the elderly feel more involved, so as to meet the mental needs of the elderly in communication, seeing and being watched, and information acquisition.

\subsection{Promote the health of the elderly}

This paper divides the subsidiary green space in the residential area into static activity area and dynamic activity area. The elderly can exercise in the dynamic activity area. The elderly in the dynamic activity area can warm up with exercise, and special exercise fitness equipment is set in the dynamic activity area to help the elderly relax their muscles and bones and prevent sports injuries. The ball game field in the dynamic activity area of the green space attached to the residential area is a sports venue for the elderly who like ball games. At the same time, corresponding games can be held to allow the elderly to actively participate in it, improve the mood of the elderly, and improve the elderly. Improve physical fitness and reduce the incidence of senile diseases. In the sports activity area, health care workers for the elderly can be arranged according to the situation, and the promotion is mainly to guide the elderly to self-care and self-physical therapy to promote the health of the elderly.

\subsection{Rehabilitation and recuperation functions of the attached green space in the residential area}

The green space attached to the residential area also has the function of rehabilitation. Compared with ordinary landscapes, the rehabilitation landscape is more special, and the rehabilitation landscape is based on rehabilitation from beginning to end. It adopts the principles of TCM health preservation and has medicinal plants in the space [11]. When the elderly are relaxing and entertaining, they are integrated into the environment created by medicinal plants to achieve the effect of restoring the body and mind and maintaining health. The medicinal plants in the green space attached to the residential area are mainly olfactory medicinal plants. When the elderly walk in the green space, they breathe into the nose and enter their bodies to achieve healing.

\section{Conclusion}

As the problem of aging in our country continues to intensify, more and more people are beginning to pay attention to the problem of aging. The elderly population continues to increase, and the issue of venues for the elderly needs to be resolved. The ancillary green space in the residential area will become the main place for the elderly, and the planning and design of the ancillary green space in the residential area is also an important issue. In the planning and design process of residential area ancillary green space, it is necessary to consider not only the scope of services, but also the activity preferences of the elderly. It is necessary to propose corresponding solutions to the problems of the elderly to better construct the residential area ancillary green space. The ancillary green space in the residential area is fully functional. From the perspective of the activity needs of the elderly, the article deepens the zoning planning layout and design practice research of the auxiliary green space in the residential area, and concludes that the scientific and reasonable residential area auxiliary green space construction can improve the living standards of the elderly and guide the healthy lifestyle of the elderly. Provide certain theoretical support for the planning and design of subsidiary green spaces in residential areas in the future.

\section{Acknowledgments}

I would like to take this opportunity to thank a number of people who have offered invaluable assistance in the preparation of the dissertation. I would like to express my heartfelt gratitude to the professors and teachers of all the College of Architecture and Art. Their insights are fully prepared for my completion of this paper.

\section{References}

1. Mu Guangzong, Zhang Tuan. The development trend of my country's population aging and its strategic response[J]. Journal of Central China Normal University (Humanities and Social Sciences Edition), 2011, 50 (5): 29-36.

2. Yang Laili. Urban garden and green space planning[M]. 3rd edition. Beijing: China Forestry Publishing House, 2012:211-212.

3. Wu Yiqu. Landscape design of residential areas based on the mental needs of the elderly[J]. Journal of Wuhan University of Technology, 2010, 32 (15) :5659.

4. Shen Shan, Hu Tinghao, Luan Ashi. Research progress of domestic urban public service facilities for the elderly[J]. Modern City Research, 2014 (9):26-33.

5. Yang Ting. Discussion on the comprehensive park design based on the behavioral psychology of the 
elderly[D]. Changsha: Central South University of Forestry and Technology, 2012.

6. Zhou Jie, Chai Yanwei. Research progress in spatial behavior of the elderly in China[J]. Advances in Geographical Sciences, 2013 (5):722-732.

7. Wei Lishu. Analysis of the humanized design of the activity space for the elderly in the garden[J]. Fujian Forestry Science and Technology, 2004, 31 (3): 7983.

8. Wang Like, Tang Jingjing. Research on the strategy of garden design based on the aging society $[\mathrm{C}] / /$ Chinese Society of Landscape Architecture, 2013 Annual Conference Proceedings (Volume 2), 2013.

9. Zhang Yunji, Pu Yongji. Research on the color configuration of green space favored by the elderly $[\mathrm{J}]$. Chinese Garden, 2009, 25 (7) :78-81.

10. Mao Yan. Discussion on setting up activity areas for the elderly in urban green spaces [J]. Chinese Garden, 1999 (4): 42-43.

11. Zhang Wenying, Feng Xiliang. The significance of inclusive design to the construction of public space in an aging society[J]. Chinese Landscape Architecture, 2012, 28 (10) :30-35. 